\title{
Prevalence of Fatigue and Associated Factors in a Spinal Cord Injury Population: Data from an Internet-Based and Face-to-Face Surveys
}

Javier Cudeiro-Blanco, ${ }^{1}$ Ana Onate-Figuérez, ${ }^{1,2}$ Vanesa Soto-León, ${ }^{1}$ Juan AvendañoCoy, ${ }^{2}$ Laura Mordillo-Mateos, ${ }^{1}$ Angela Brocalero-Camacho, ${ }^{1}$ Ana Esclarin-Ruz, ${ }^{3}$ Mario Rotondi, ${ }^{4}$ Juan Aguilar, ${ }^{5}$ Pablo Arias, ${ }^{6}$ and Antonio Oliviero ${ }^{1}$

AuthorAffiliation

${ }^{1}$ FENNSI Group, Hospital Nacional de Parapléjicos, SESCAM, Toledo, Spain.

${ }^{2}$ GIFTO Group, E. U. de Enfermia y Fisioterapia de Toledo, Universidad de Castilla La Mancha, Toledo, Spain.

${ }^{3}$ Rehabilitation Department, Hospital Nacional de Parapléjicos, SESCAM, Toledo, Spain.

${ }^{4}$ Unit of Internal Medicine and Endocrinology, ICS-Maugeri I. R. C. C. S. , Laboratory for Endocrine Disruptors and University of Pavia, Pavia, Italy.

${ }^{5}$ Experimental Neurophysiology Group, Hospital Nacional de Parapléjicos, SESCAM, Toledo, Spain.

6 NEUROcom. Neuroscience and Motor Control Group, Department of Medicine-INEF-INIBIC, University of Coruña, Coruña, Spain.

Both authors contributed equally to this article.

Address correspondence to: Antonio Oliviero, FENNSI Group, Hospital Nacional de Parapléjicos, Finca La Peraleda s/n 4, 5071 Toledo, Spain, E-mail: antonioo@sescam.jccm.es 


\begin{abstract}
Fatigue has a profound impact on patients with spinal cord injury (SCI), but only limited treatments are available. The aim of this study was to determine the prevalence of fatigue in SCI and its association with clinical and demographic factors. We used an internet-based survey and a face-to-face interview to estimate the prevalence of fatigue in a SCI population. Fatigue was measured using the Fatigue Severity Scale (FSS). Clinically significant fatigue was defined as FSS scores greater than or equal to four. A total of 253 participants with SCI were included in the study. Clinically significant fatigue was present in one third of our sample. There was no relationship between fatigue and injury level or completeness. We found significant correlations between depression, pain, and level of injury. The relation of fatigue with completeness of injury and spasticity is less clear. Moreover, the online survey and the standard face-to-face interview showed similar results concerning fatigue evaluation. Several factors may contribute to fatigue, however. Future studies should be conducted to clarify which are the most relevant ones and, if possible, to determine which factors are modifiable.
\end{abstract}

Keywords: ASIA; fatigue; SCI; tetraplegia 


\section{Introduction}

Spinal cord Injury (SCI) has a great impact on the quality of life of the affected patients. ${ }^{1,2}$ Further, unhealthy lifestyle status has been shown to positively correlate to high levels of psychological fatigue. ${ }^{3}$ Fatigue is a common symptom in patients with SCI, ${ }^{3-6}$ and it refers to a state of either mental and physical tiredness or exhaustion. A limited number of specific treatments are available, and, moreover, fatigue is often undermentioned and/or underestimated in medical interviews in the SCI population. ${ }^{5,7}$ Fatigue is a universal human experience, and it diminishes the capacity of those with it to conduct activities of daily living (such as working and even maintaining personal relationships). ${ }^{4-6,8}$ Fatigue has generally been referred to as a change in performance of a task over time because of both physiological and psychological factors. ${ }^{9}$ Fatigue should be distinguished from sleepiness, which refers to the probability of falling asleep, and from physiological tiredness proportional to the performed physical or mental activity. ${ }^{9}$ The etiology of fatigue in central nervous system disease is poorly understood. Further, fatigue is often difficult to measure because it can change over time.

In SCI, fatigue can be because of several factors such as patient medication, chronic pain, spasticity, autonomic dysfunction, behavioral factors, increased energy costs associated with functioning, sleep disorders, and psychiatric disturbance (i.e., depression). ${ }^{3,8,10-12}$ Moreover, a strong relationship between pain, depressive mood, and fatigue has been reported in SCI. ${ }^{13}$ Fatigue may also arise after SCI because the central nervous system fails to adequately drive the spinal motoneurons (central fatigue) ${ }^{14-}$

${ }^{16}$ Recently, the course of fatigue after SCI has been reported to remain stable during rehabilitation and after discharge into the community. ${ }^{17}$ These authors also suggested that fatigue assessment should be repeated over time throughout the rehabilitation process. ${ }^{17}$

The Fatigue Severity Score (FSS) is a method for measuring fatigue that was originally developed for those with multiple sclerosis. ${ }^{18}$ It is also suitable for SCI populations. ${ }^{6}$ Moreover, the FSS is a questionnaire that is suitable for an internet-based survey, thus allowing a large population to be studied in a short time and in an inexpensive manner. ${ }^{18}$ To the best of our knowledge, a comparison between internet- 
based and face-to-face surveys has never been performed to evaluate clinically significant fatigue in a SCI population study.

The main aim of this study was to evaluate the prevalence of clinically significant fatigue by using two different approaches: an internet-based survey and a face-to-face interview. If the two approaches yielded similar results, it could be proposed that administering the FSS online would provide a reliable clinical tool in patients with SCI. As a secondary aim, the association between clinically significant fatigue and demographic and clinical factors in SCI was also analyzed and reported.

\section{Methods}

Participants and recruitment

Data were obtained from two different sources: an online survey and face-to-face interviews.

\section{Online survey}

Two hundred and fourteen consecutive participants were recruited over six weeks using Survey Monkey, a Web 2.0 platform. They received a participant information sheet, an electronic consent indicator, and the survey itself. Only SCI participants who were able to complete all the items of the FSS questionnaire were included in the data set.

Face-to-face survey

Seventy-three unselected, consecutive participants were recruited over a total period of eight weeks at the Hospital Nacional de Parapléjicos of Toledo, Spain. The 73 participants were interviewed personally, and their answers were collected in paper format. All of them successfully completed the entire survey. Subjects that participated in the face-to-face survey were not allowed to participate in the online survey. 


\section{Inclusion criteria}

Inclusion criteria were a diagnosis of SCI, the ability to provide informed consent, being between 16 and 80 years old, and able to answer all the questions of the FSS.

The local ethical committee (CEIC-Toledo, Spain) approved the study, which was performed accordingly to the declaration of Helsinki. All the participants gave their informed consent to participate to the study.

\section{Data collection}

The online survey consisted of 31 questions in total, and it took approximately $10 \mathrm{~min}$ to be completed. The survey was divided in two parts. During the first one, contact information, demographic data, and self-reported data for disease profile were collected (e.g., age, sex, lesion level, etc.).Then, participants were asked to leave an optional comment regarding their previous information before continuing with the second part. The second part contained the FSS questionnaire.

On some occasions, participants did not have complete knowledge of their disease profile (e.g., American Spinal Injury Association [ASIA]), yet they were included in the study. Only those participants who did not answer all the questions of the FSS were not included in the dataset (exclusion criteria).

The face-to-face interview was more comprehensive. It included additional questions about sociodemographic factors (e.g., alcohol, tobacco, or marijuana habits), and a more detailed evaluation of the patient's clinical history (e.g., urinary tract dysfunction). It took approximately $20 \mathrm{~min}$ to complete. 


\section{Demographic data}

Demographic items assessed in both surveys were: sex, age, and educational level. In the online survey, age was recorded in five specific ranges $(18-29,30-39,40-49,50-59$, and 60+). Three educational levels were considered that approximately correspond to primary school (0-6 years of education), secondary school (7-12 years), and university studies (more than 12 years).

\section{Clinical data}

Both surveys conveyed questions about the following clinical data: cause of the lesion (traumatic or not), completeness of the lesion (complete or incomplete), ASIA impairment scale (A to E), level of SCI (cervical, thoracic, or lumbar), and time since SCI lesion. In the online survey, time since lesion was recorded in four specific ranges (less than 1 year, 1-3 years, 3-10 years, and more than 10 years). The ASIA impairment scale was used to confirm the completeness or incompleteness of the lesion (reported by the participants). Moreover, both surveys contained questions about self-assessment of: walking abilities ("autonomous," "can walk with assistance," or "not able to walk"), pain ("yes" or "no"), spasticity ("yes" or "no") and depression ("yes" or "no").

Pain and spasticity were also graded. Pain was graded asking the subject to rate the pain from 0 , no pain, and 10, maximum pain. Spasticity was graded by asking the subject to rate the spasticity as "no spasticity," "mild," "moderate," "severe," and "very severe." We will refer to this latter variable as "severity of spasticity." As far as walking abilities are concerned, participants reporting "can walk with assistance" included both therapeutic walking (for rehabilitation purpose) and free walking with help of a person or devices. 


\section{Fatigue evaluation}

The FSS is arguably the most widely used questionnaire to assess fatigue severity in neurological disorders, and it is easy to use both in clinical practice and research. ${ }^{18-23}$ It was designed to measure both fatigue and the effects of fatigue on function, which makes its use in rehabilitation settings particularly appealing. The FSS was administered in both surveys to assess fatigue. The FSS is a widely validated tool comprising nine fatigue-related statements that are rated on a seven-point scale (disagree to agree). ${ }^{14}$ It has good internal consistency, stability, sensitivity to change over time, and it has been used in SCI internationally. ${ }^{2,4,5}$ The criteria used to determine clinically significant fatigue was a mean score greater than or equal to four in the FSS. $^{7}$ To derive this summary score, full item completion was required. Both online and face-to-face surveys included the exact same FSS questionnaire. The only difference between them was the presence of the interviewer in the latter one.

\section{Statistics}

Sample size. There were no previous studies that compared the estimation of fatigue prevalence or incidence using two different survey approaches (online and face-to-face) in SCI or in any similar population. We were therefore unable to perform an a priori power calculation for our study. We instead chose a sample size that was similar to other studies ${ }^{5,17}$ for the face-to-face survey. We aimed for a sample size of 60 and planned to oversample by $20 \%$. For the online survey, we chose as a minimum number of participants to be surveyed the same as for the face-to face-survey, but we let the recruitment open until the data analysis started.

Data preparation. The variables "age" and "time since injury" were collected in different formats. In the face-to-face survey, these variables were saved as exact numbers (e.g., 6 months, 5 years, etc.), whereas in the online survey, they were given in specific ranges (e.g., 1-5 years, older than age 60). The numerical information from the face-to-face survey was transformed to fit within the same ranges of the online survey. 
Data analysis. Participants with SCI were stratified according to the presence (FSS $\geq 4$ ) or absence (FSS <4) of clinically significant fatigue. To identify potential differences determined by the kind of survey for the assessment of clinically significant fatigue, the type of survey was considered as a dichotomous variable. Thus, a first binary logistic regression model was constructed. In this first model, the type of survey was entered as the dependent variable while age, sex, cause, completeness, lesion level, time since injury, educational level, and significant fatigue (FSS >4) all served as covariates. Because the results showed that there was a nonsignificant influence of the type of survey on the presence of significant fatigue (FSS $\geq 4$ ), participants from the two surveys were pooled as a whole group.

The pooled data were entered in a second binary logistic regression model constructed by entering significant fatigue (FSS >4 and FSS <4) as the dependent variable and age, sex, cause, completeness, lesion level, time since injury, educational level, walking ability, pain, spasticity, and depression as covariates. This second model allowed identifying all variables significantly related to the presence of clinically significant fatigue. The variables were further compared between the two groups of participants characterized by an FSS $\geq 4$ and FSS $<4$. A Mann-Whitney U test was used to compare the nonparametric variables. The Spearman rho test was used to analyze the correlation between these variables and the FSS score. The significance level was set at $p \leq 0.05$.

\section{Results}

A total of 214 SCI participants participated in the online survey, but 34 did not complete the FSS, so only 180 were finally included in the study. Thus, a total of 253 persons with SCI were included in the study (180 from the online survey and 73 from the face-to-face survey). Clinically significant fatigue (FSS $\geq 4$ ) was found in 94 of 253 subjects $(37.2 \%$ of the total number of participants). Divided by groups, significant fatigue was found in $28.8 \%$ of subjects in the face-to-face survey, and $40.6 \%$ in the online survey (Table 1). Regarding the online survey, only 95 of 180 participants (53\%) reported their ASIA impairment scale. All the participants who reported ASIA A reported to have a "complete" lesion, and all the participants reporting ASIA C, D, and 
E declared to have an "incomplete" lesion. On the other hand, all the participants reporting ASIA B but two declared to have an "incomplete lesion." In these two cases, we elected to consider the answer to the completeness reporting so these two participants were included in the analysis as "complete."

\section{Online and face-to-face surveys}

The results of the logistic regression demonstrated a significant association between time since injury (odds ratio $[\mathrm{OR}]=0.101$, confidence interval $[\mathrm{CI}]=0.055-0.185 ; \mathrm{p}<$ $0.001)$ and educational level $(\mathrm{OR}=0.612, \mathrm{CI}=0.394-0.950 ; \mathrm{p}=0.029)$ with survey (Table 1). These variables were independently and significantly related to survey, as demonstrated by the lack of any overlap between the confidence intervals in the multivariate analysis (Table 2). The two most evident differences between the two surveys were the time since lesion, which was shorter in the face-to-face survey, and the educational level, which was higher in the online survey. There was no difference in the clinically significant fatigue between the two surveys. Because we did not find differences in the clinically significant fatigue between the two surveys, the data were pooled across studies, and a second binary logistic regression model was constructed. The sociodemographic and clinical data of SCI participants with and without clinically significant fatigue are reported in Table 1.

The results of our second logistic regression demonstrated a significant association between lesion level, pain, and depression with fatigue (Table 3). Lesion level, pain, and depression were significantly related with fatigue, but they were not completely independent variables, as demonstrated by the overlap between the confidence intervals in the multi-variate analysis (Table 3). Patient with lesions at a cervical level showed significantly more fatigue (Mann-Whitney $U$ test, $Z=-2.102 ; \mathrm{p}=0.036$ ) compared with those with lumbar and thoracic lesions. Participants with depression were significantly more fatigued (Mann-Whitney $\mathrm{U}$ test, $\mathrm{Z}=-4.872$; $\mathrm{p}<0.0001$ ). Participants with pain had significantly more clinically significant fatigue (Mann-Whitney $U$ test, $Z=-2.815$; $\mathrm{p}<0.01$ ), and fatigue was positively correlated with pain severity (Spearman rho test, rho $=0.351 ; \mathrm{p}<0.0001)$ (Fig. 1). 
FIG. 1. (A) shows the Fatigue Severity Scale (FSS) values of patients with spinal cord injury (SCI) with different lesion levels. Cervical patients have significantly more fatigue $(Z=-2.102 ; p=0.036)$ compared with those with lumbar and thoracic lesions. (B) shows the FSS values of patients with and without depression. Patients with depression have significantly more fatigue $(Z=-4.872 ; \mathrm{p}<0.0001)$. (C) shows the FSS values of patients with and without pain. Patients with pain have significantly more fatigue $(Z=-2.815 ; p<0.01)$. (D) shows the FSS values of SCI patients with and without pain. Fatigue is positively correlated with pain (rho $=0.351 ; p<0.0001)$. ${ }^{*} p<$ 0.05 .

\section{Association of spasticity or lesion incompleteness with fatigue}

A box plot of these two variables against FSS is presented (Fig. 2). First, participants with incomplete lesions were found to have a tendency to have more fatigue than those with complete lesions (Mann-Whitney $U$ test, $Z=-1.877 ; p=0.061$ ). Second, there was no difference between participants with and without spasticity in terms of fatigue. There was a correlation, however, between fatigue and the severity of spasticity (Spearman rho test, rho $=0.160 ; p=0.011)$. This suggests that fatigue only increases in participants with higher degrees of spasticity. Consumption of alcohol, tobacco, and marijuana had no correlation with fatigue. Urinary tract dysfunction did not show any relationship with fatigue either.

FIG. 2. (A) shows the Fatigue Severity Scale (FSS) values of patients with spinal cord injury (SCI) with complete or incomplete lesions. Our data showed a tendency for incomplete patients to have more fatigue than complete patients $(\#=0.061)$. (B) shows that the FSS values of SCI patients with or without spasticity are similar. (C) shows the FSS values of SCI patients with different spasticity severities. These data suggest that patients with more severe spasticity have more fatigue (rho $=0.160 ; \mathrm{p}=0.011$ ). 


\section{Discussion}

This study provides an analysis of the different associations of demographic and clinical variables with clinically significant fatigue in SCI participants. Approximately one third of those who participated in this study showed clinically significant fatigue, confirming the impact that this condition has in SCI. ${ }^{5-7,17,22}$ To evaluate fatigue, the FSS questionnaire was chosen, because it is a widely used and validated tool in the clinic, it is easy to assess, and it has been well studied in other disorders ${ }^{19-23}$ Moreover, it is possible to conduct this survey online. ${ }^{24}$

A relevant difference in clinically significant fatigue was associated with the lesion level. The proportion of cervical patients with fatigue was significantly higher than patients with lumbar and thoracic lesions. This may not be surprising, because cervical lesions are associated with worse motor function (taking into account the severity) and with more severe autonomic dysfunctions. On the other hand, lesion level is not a fully independent variable, because it is, at least, associated with depression and pain. So it is possible that worse motor function and autonomic dysfunction play a role in generating fatigue, but it is also possible that this is because of a higher prevalence of depression and pain in patients with cervical lesions. ${ }^{24,25}$

Two studies reported more evident fatigue in patients with incomplete compared with patients with complete SCI. ${ }^{6,22}$ Other authors, ${ }^{17}$ however, did not confirm this in patients with purely traumatic SCI. We have shown than patients with SCI who have incomplete lesions may have more fatigue than those with complete lesions, in a group of patients with lesions that include those of both traumatic and nontraumatic etiology (the difference did not reach statistical significance). These data should be confirmed in a larger sample.

Clinically significant fatigue was also significantly prevalent in patients with SCI who had depression and pain. Several studies have reported a positive association between pain and fatigue in SCI, and both pain and fatigue were negatively associated with physical functioning and mood ${ }^{8,10-12}$; our results support such observations. Our data did not allow, however, for the identification of a causal link between these two 
symptoms and fatigue. Regarding spasticity, we had expected to find more significant fatigue in patients with spasticity than in patients without it. We found, however, that only patients with a higher degree of spasticity had more clinically significant fatigue.

We showed that the online survey had minimum differences compared with the standard face-to-face interview. Moreover, there were no differences in the evaluation of clinically significant fatigue in the two surveys. Therefore, online surveys can be a useful tool to obtain relevant and reliable information from patients. It is necessary to underline, however, a few differences between the two surveys. The two most evident differences were that the time since lesion was shorter in the participants of the face-toface survey, and that the educational level was higher in the participants of the online survey. These differences can be explained by the fact that participants in the face-toface survey included some patients who were still completing their rehabilitation programs in the hospital as inpatients. Moreover, patients who underwent the online survey had access to more advanced technologies and were possibly more educated. The results obtained from the two surveys are congruent with those of others reporting on fatigue in SCI, which suggests a high level of robustness in our data. ${ }^{5-8,10-12}$

\section{Study limitations}

This study was strengthened by a large sample of patients with SCI who had a broad spectrum of disability levels and backgrounds. Our data, however, may have been affected by responder bias, because all data collected for this study were self-reported. Therefore, there is a possibility of measurement error because of self-report answers, which may bias the results because of dependent misclassification. It is possible to argue that the online data may have been more affected by responder bias (e.g., in the face-to-face survey, the participants may ask for an explanation for questions they cannot understand). On the other hand, this seems unlikely in view of the similar rate of fatigue recorded when the two surveys were analyzed separately. Further, we investigated the impact of pain, spasticity, and depression on fatigue, but we cannot exclude that the pharmacological treatments, and not the symptoms themselves, may have a relation with clinically significant fatigue. 


\section{Conclusion}

We found that clinically significant fatigue is frequent in SCI, and it is present in one third of the patients. We encourage clinicians not to underestimate this symptom, because it can have functional impact on patients with SCI. We demonstrated that internet-based survey results are similar to those of the face-to-face survey. Thus, we suggest that an internet-based survey can be a useful tool to administrate the FSS easily, and this can facilitate the evaluation and follow-up of patients with SCI. Further, because many factors contribute to fatigue, future studies will be conducted to clarify which are the most relevant ones and, if possible, to determine which factors are modifiable.

\section{Acknowledgments}

We would like to thank Dr. Bryan A. Strange for language editing.

\section{Author Disclosure Statement}

No competing financial interests exist.

\section{References}

1. Wyndaele M. and Wyndaele J.J. (2006). Incidence, prevalence and epidemiology of spinal cord injury: what learns a worldwide literature survey? Spinal Cord 44, 523 - 529.

2. Jensen M.P., Molton I.R., Groah S.L., Campbell M.L., Charlifue S., Chiodo A., Forchheimer M., Krause J.S., and Tate D. (2012). Secondary health conditions in individuals aging with SCI: terminology, concepts and analytic approaches. Spinal Cord 50, 373 - 378.

3. Craig A., Tran Y., Wijesuriya N., and Middleton J. (2012). Fatigue and tiredness in people with spinal cord injury. J. Psychosom. Res. 73, 205 - 210.

4. McKinley W.O., Jackson A.B., Cardenas D.D., and DeVivo M.J. (1999). Long-term medical complications after traumatic spinal cord injury: a regional model systems analysis. Arch. Phys. Med. Rehabil. 80, 1402 - 1410.

5. Anton H.A., Miller W.C., and Townson A.F. (2008). Measuring fatigue in persons with spinal cord injury. Arch. Phys. Med. Rehabil. 89, 538 - 542. 
6. Fawkes-Kirby T.M., Wheeler M.A., Anton H.A., Miller W.C., Townson A.F., and Weeks C.A. (2008). Clinical correlates of fatigue in spinal cord injury. Spinal Cord 46, 21 - 25.

7. Lee A.K., Miller W.C., Townson A.F., and Anton H.A. (2010). Medication use is associated with fatigue in a sample of community-living individuals who have a spinal cord injury: a chart review. Spinal Cord 48, 429 - 433.

8. Hammell K.W., Miller W.C., Forwell S.J., Forman B.E., and Jacobsen B.A. (2009). Fatigue and spinal cord injury: a qualitative analysis. Spinal Cord 47, 44 - 49.

9. Craig A, Tran Y, Wijesuriya N, and Boord P. (2006). A controlled investigation into the psychological determinants of fatigue. Biol. Psychol. 72, 78 - 87.

10. Freixes O., Rivas M.E., Agrati P.E., Bochkezanian V., Waldman S.V., and Olmos L.E. (2012). Fatigue level in spinal cord injury AIS D community ambulatory subjects. Spinal Cord 50, 422 425.

11. Saunders L.L. and Krause J.S. (2012). Behavioral factors related to fatigue among persons with spinal cord injury. Arch. Phys. Med. Rehabil. 93, 313 - 318.

12. Barat M., Dehail P., and de Seze M. Fatigue after spinal cord injury. (2006). Ann. Readapt. Med. Phys. 49, 365 - 369.

13. Craig A., Tran Y., Siddall P., Wijesuriya N., Lovas J., Bartrop R., and Middleton J. (2013). Developing a model of associations between chronic pain, depressive mood, chronic fatigue, and self-efficacy in people with spinal cord injury. J. Pain 14, 911 - 920.

14. Gandevia S.C. Spinal and supraspinal factors in human muscle fatigue. (2001). Physiol. Rev. 81, $1725-1789$.

15. Arias P., Robles-Garcia V., Corral-Bergantinos Y., Madrid A., Espinosa N., Valls-Sole J., Grieve K.L., Oliviero A., and Cudeiro J. (2015). Central fatigue induced by short-lasting finger tapping and isometric tasks: a study of silent periods evoked at spinal and supraspinal levels. Neuroscience 305, $316-327$.

16. Madrid A., Valls-Sole J., Oliviero A., Cudeiro J., and Arias P. (2016). Differential responses of spinal motoneurons to fatigue induced by short-lasting repetitive and isometric tasks. Neuroscience $339,655-666$.

17. Anton H.A., Miller W.C., Townson A.F., Imam B., Silverberg N., and Forwell S. (2017). The course of fatigue after acute spinal cord injury. Spinal Cord 55, 94 - 97.

18. Krupp L.B., LaRocca N.G., Muir-Nash J., and Alfred D. Steinberg A.D. (1989). The fatigue severity scale. Application to patients with multiple sclerosis and systemic lupus erythematosus. Arch. Neurol. 46, 1121 - 1123.

19. Dittner A.J., Wessely S.C., and Brown R.G. (2004). The assessment of fatigue: a practical guide for clinicians and researchers. J. Psychosom. Res. 56, 157 - 170.

20. Herlofson K. and Larsen J.P. (2002). Measuring fatigue in patients with Parkinson's disease--the Fatigue Severity Scale. Eur. J. Neurol. 9, 595 - 600.

21. Schepers V.P., Visser-Meily A.M., Ketelaar M., and Lindeman E. (2006). Poststroke fatigue: course and its relation to personal and stroke-related factors. Arch. Phys. Med. Rehabil. 87, 184 - 188. 
22. Nooijen C.F., Vogels S., Bongers-Janssen H.M., Bergen M.P., Stam H.J., and van den Berg-Emons H.J. (2015). Fatigue in persons with subacute spinal cord injury who are dependent on a manual wheelchair. Spinal Cord 53, 758 - 762.

23. LaChapelle D.L. and Finlayson M.A. (1998). An evaluation of subjective and objective measures of fatigue in patients with brain injury and healthy controls. Brain Inj 12, 649 - 659.

24. Weiland T.J., Jelinek G.A., Marck C.H., Hadgkiss E.J., van der Meer D.M., Pereira N.G., et al. (2015). Clinically significant fatigue: prevalence and associated factors in an international sample of adults with multiple sclerosis recruited via the internet. PLoS One 10, e0115541.

25. Khazaeipour Z., Taheri-Otaghsara S.M., and Naghdi M. (2015). Depression following spinal cord injury: its relationship to demographic and socioeconomic indicators. Top. Spinal Cord Inj. Rehabil. $21,149-155$. 
TABLE 1. PATIENT DEMOGRAPHICS

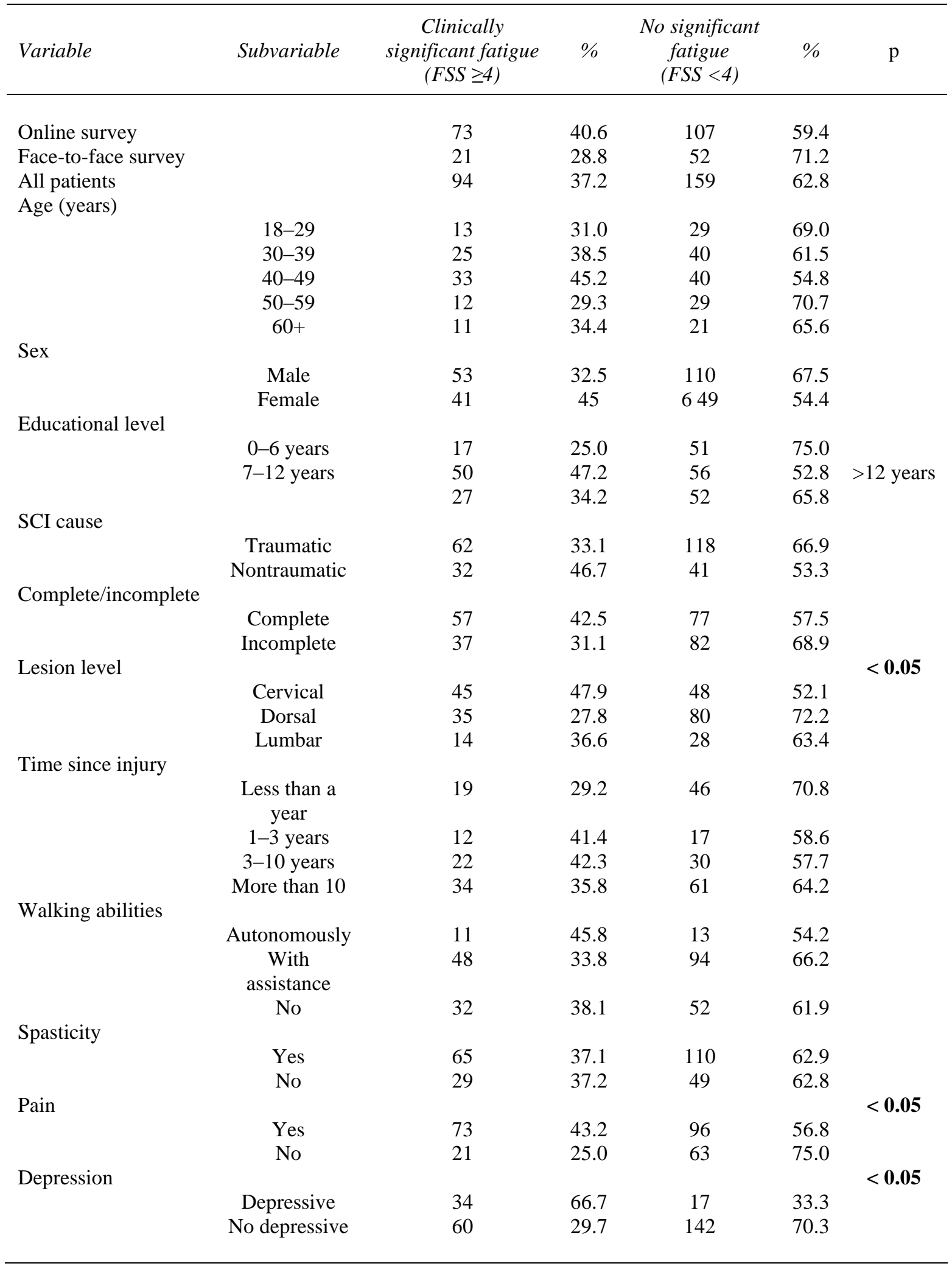

FSS, Fatigue Severity Scale; SCI, spinal cord injury 
TABLE 2

\begin{tabular}{lcccc}
\hline Variable & OR & Sig. & 95\% CL Lower & 95\% CL Upper \\
\hline & & & & \\
Age & 1.125 & 0.583 & 0.739 & 1.712 \\
Sex & 1.358 & 0.577 & 0.464 & 3.972 \\
Cause & 0.371 & 0.126 & 0.104 & 1.32 \\
Completeness & 0.84 & 0.753 & 0.282 & 2.499 \\
Level & 0.905 & 0.787 & 0.439 & 1.867 \\
Time since injury & 0.101 & $\mathbf{0 . 0 0 0}$ & 0.055 & 0.185 \\
Education & 0.612 & $\mathbf{0 . 0 2 9}$ & 0.394 & 0.95 \\
Significant fatigue & 0.61 & 0.368 & 0.208 & 1.789 \\
& & & & \\
\hline
\end{tabular}

OR, odds ratio; CL, confidence limits.

TABLE 3

\begin{tabular}{lcccc}
\hline Variable & OR & Sig. & 95\% CL Lower & 95\% CL Upper \\
\hline & & & & \\
Age & 0.968 & 0.805 & 0.747 & 1.253 \\
Sex & 0.651 & 0.176 & 0.349 & 1.212 \\
Cause & 1.497 & 0.272 & 0.729 & 3.076 \\
Completeness & 1.499 & 0.227 & 0.777 & 2.894 \\
Lesion level & 1.679 & $\mathbf{0 . 0 2 7}$ & 1.062 & 2.654 \\
Time since injury & 1.076 & 0.597 & 0.82 & 1.41 \\
Education & 1.039 & 0.783 & 0.792 & 1.362 \\
Walking ability & 0.984 & 0.961 & 0.525 & 1.846 \\
Depression & 4.650 & $\mathbf{0 . 0 0 0}$ & 2.23 & 9.696 \\
Pain & 2.191 & $\mathbf{0 . 0 2 3}$ & 1.117 & 4.298 \\
Spasticity & 0.769 & 0.455 & 0.386 & 1.533 \\
\end{tabular}

OR, odds ratio; CL, confidence limits. 

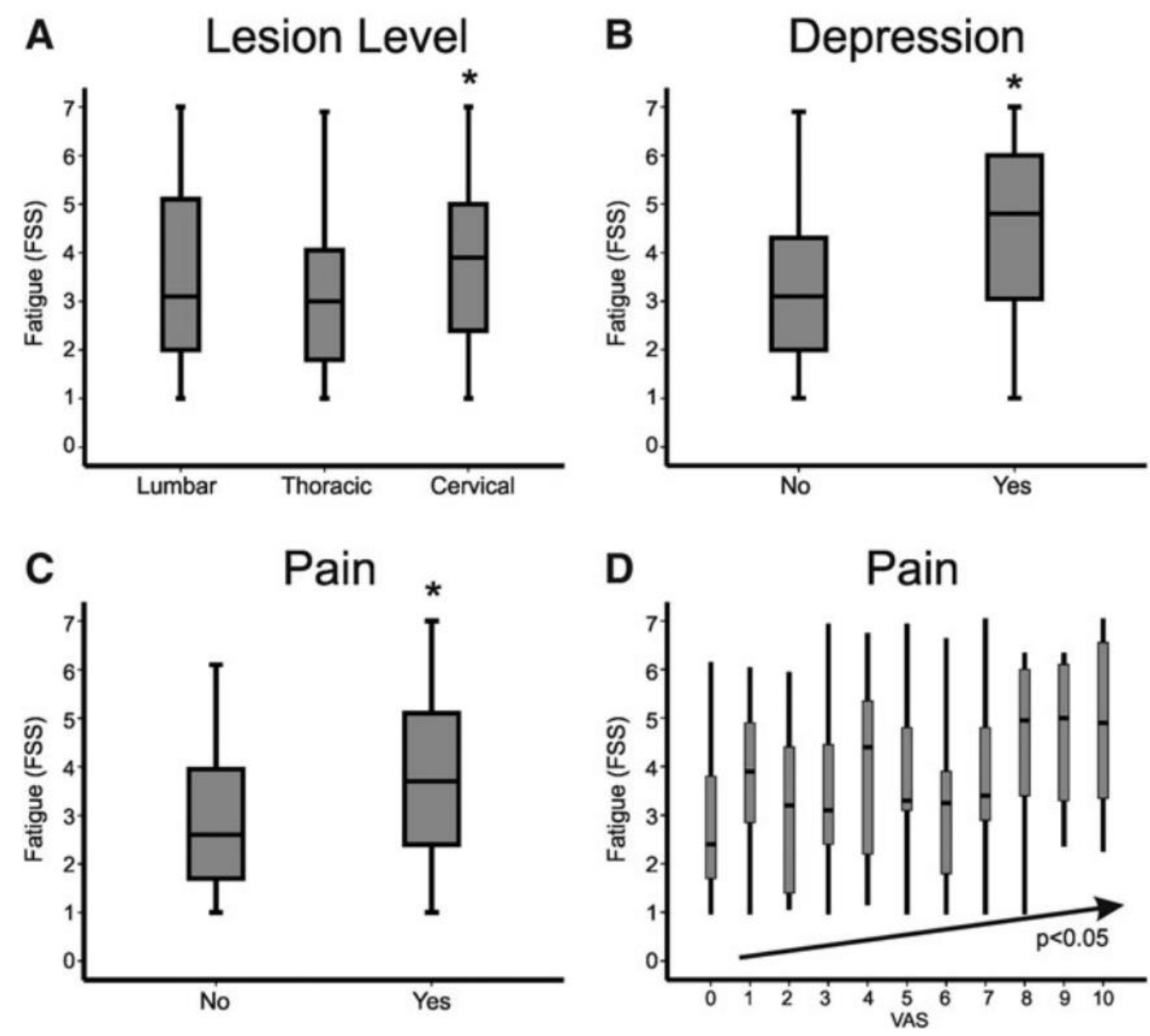

FIG. 1. (A) shows the Fatigue Severity Scale (FSS) values of patients with spinal cord injury (SCI) with different lesion levels. Cervical patients have significantly more fatigue $(\mathrm{Z}=-2.102 ; p=0.036)$ compared with those with lumbar and thoracic lesions. (B) shows the FSS values of patients with and without depression. Patients with depression have significantly more fatigue $(\mathrm{Z}=-4.872 ; p<0.0001)$. (C) shows the FSS values of patients with and without pain. Patients with pain have significantly more fatigue $(\mathrm{Z}=$ $2.815 ; p<0.01$ ). (D) shows the FSS values of SCI patients with and without pain. Fatigue is positively correlated with pain $($ rho $=0.351 ; p<0.0001) . * p<0.05$. 


\section{A Completeness}

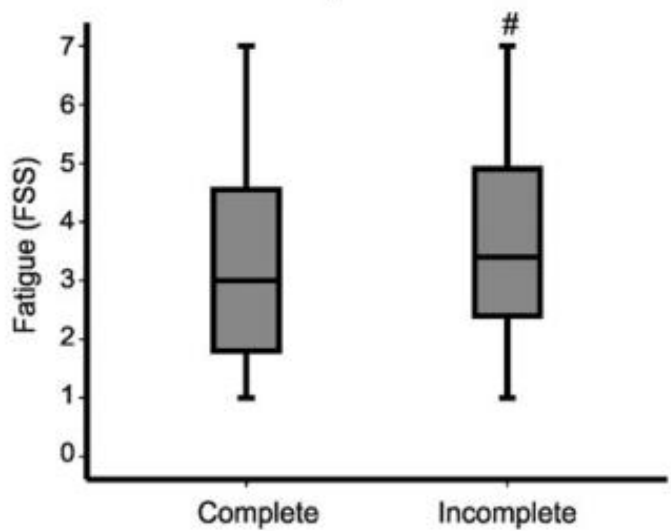

B Spasticity
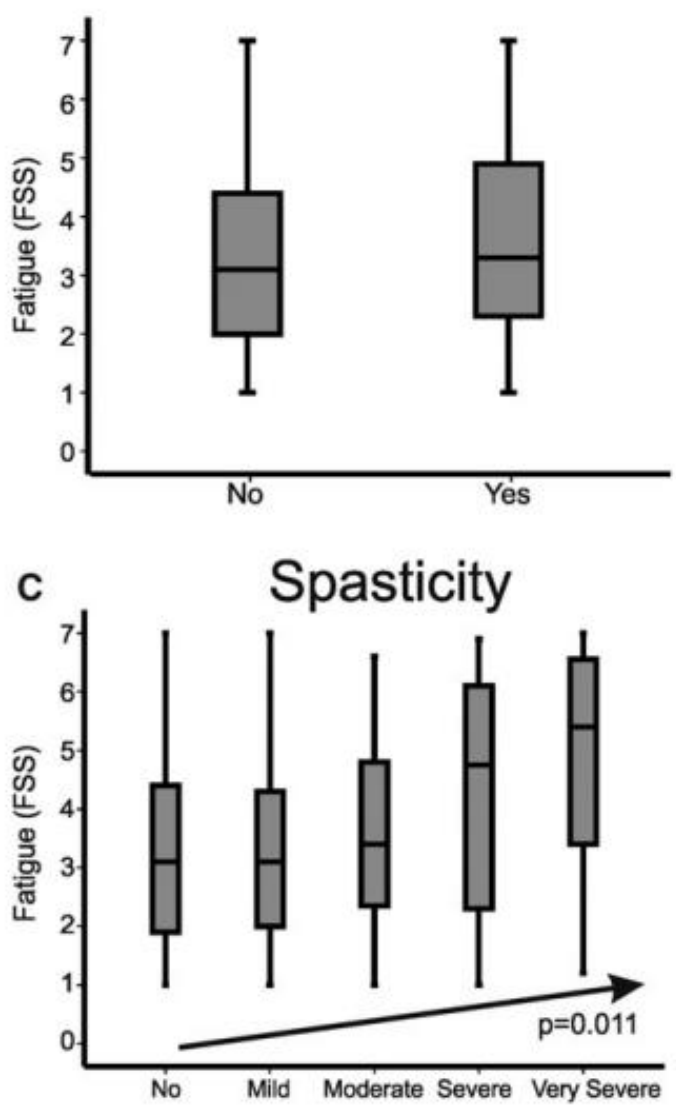

FIG. 2. (A) shows the Fatigue Severity Scale (FSS) values of patients with spinal cord injury (SCI) with complete or incomplete lesions. Our data showed a tendency for incomplete patients to have more fatigue than complete patients $(\#=0.061)$. (B) shows that the FSS values of SCI patients with or without spasticity are similar. (C) shows the FSS values of SCI patients with different spasticity severities. These data suggest that patients with more severe spasticity have more fatigue (rho $=0.160 ; p=0.011$ ). 